APCTP 97-05

PM 97-05

March 1997

\title{
Higgs Boson Decays into Light Gravitinos
}

\author{
Abdelhak Djouadi ${ }^{1}$ and Manuel Drees ${ }^{2}$ \\ ${ }^{1}$ Laboratoire de Physique Mathématique et Théorique, UPRES-A 5032, \\ Université de Montpellier II, F-34095 Montpellier Cedex 5, France. \\ ${ }^{2}$ APCTP, 207-43 Cheongryangri-dong, Tongdaemun-gu, \\ Seoul 130-012, Korea
}

\begin{abstract}
We study decays of the neutral and charged Higgs bosons of the minimal supersymmetric standard model into a very light gravitino and a neutralino or chargino. Present experimental constraints already imply that the lighter scalar Higgs boson can only have a very small branching ratio into this mode. However, if the gravitino mass is below $\sim 0.5 \mathrm{eV}$, the heavier neutral and charged Higgs boson decays into a gravitino can be important or even dominant. We discuss the resulting signature for associate production of the heavy $\mathrm{CP}$-even and the $\mathrm{CP}$-odd states at future $e^{+} e^{-}$ colliders, and comment on Higgs boson production at hadron colliders.
\end{abstract}




\section{Introduction}

Recently models with a very light gravitino $\widetilde{G}$ [目], $m_{\widetilde{G}} \leq 10^{-3} \mathrm{eV}$, have attracted some attention [2, 3, 4]. This interest was originally triggered by the resurgence of models [5] where supersymmetry breaking is transmitted by gauge interactions to the "visible sector" containing the usual gauge, Higgs and matter superfields, although these models tend to predict $m_{\widetilde{G}} \geq 1 \mathrm{eV}$. Certain supergravity models can also naturally accommodate a very light gravitino [6].

Another motivation comes from CDF's observation [7] of a single event with an $e^{+} e^{-}$ pair, two hard photons, and missing transverse momentum. The probability for such an event to come from Standard Model (SM) processes is about $10^{-3}$, but it can quite easily be explained [8, 2] in models with a light gravitino. It should be admitted that the absence of additional events with two hard photons and missing transverse momentum [9] is beginning to cast some doubt [10] on this explanation. Nevertheless models with a very light gravitino remain an interesting field of study in their own right [1].

In this Letter we explore the impact that a very light gravitino could have on the phenomenology of the Higgs sector of the minimal supersymmetric standard model (MSSM). Higgs bosons do not couple to pairs of gravitinos, but they do couple to a gravitino and a neutralino or a chargino. The corresponding branching ratios are computed in Section 2. Since in this scenario even the lightest neutralino is unstable, $H \rightarrow \widetilde{G} \tilde{\chi}$ decays give rise to final states containing a hard photon, missing energy, and additional leptons and/or jets if $\tilde{\chi} \neq \tilde{\chi}_{1}^{0}$. Higgs search strategies may have to be modified to include such final states. One example, the associate production of the heavier $\mathrm{CP}$-even and $\mathrm{CP}$-odd neutral Higgs bosons at a future $e^{+} e^{-}$collider, is discussed in Section 3. Finally, Section 4 contains a brief summary and some conclusions.

\section{Decay branching ratios}

As first pointed out by Fayet [1], the couplings of the "longitudinal" (spin 1/2) components of the gravitino to ordinary matter are enhanced by the inverse of the gravitino mass; if $m_{\widetilde{G}}$ is sufficiently small, this can compensate the suppression by the inverse Planck mass $M_{P}=2.4 \cdot 10^{18} \mathrm{GeV}$ that appears in all gravitational interactions. In fact, a longitudinal gravitino is [1, 11] nothing but the "Goldstino" that signals the spontaneous breaking of global supersymmetry (SUSY), and whose coupling are inversely proportional to the SUSY breaking scale $\Lambda^{2} \sim m_{\widetilde{G}} M_{P}$. Since Goldstino couplings contain momenta of the external particles, partial widths for decays into final states containing (longitudinal) gravitinos depend very strongly on the mass of the decaying particle.

Specifically, for the case at hand, one has for the partial decay widths

$$
\Gamma\left(\Phi \rightarrow \tilde{\chi}_{i} \widetilde{G}\right)=\frac{1}{48 \pi} \kappa_{i \Phi} \frac{m_{\Phi}^{5}}{m_{\widetilde{G}}^{2} M_{P}^{2}}\left[1-\left(\frac{m_{\tilde{\chi}_{i}}}{m_{\Phi}}\right)^{2}\right]^{4},
$$

where $\Phi$ stands for a neutral or charged Higgs boson and $\tilde{\chi}_{i}$ is one of the four neutralinos or two charginos of the MSSM. The coupling factors $\kappa_{i \Phi}$ are given by [2]:

$$
\kappa_{i h}=\left|N_{i 3} \sin \alpha-N_{i 4} \cos \alpha\right|^{2}
$$




$$
\begin{aligned}
\kappa_{i H} & =\left|N_{i 3} \cos \alpha+N_{i 4} \sin \alpha\right|^{2} \\
\kappa_{i A} & =\left|N_{i 3} \sin \beta+N_{i 4} \cos \beta\right|^{2} \\
\kappa_{i H^{ \pm}} & =\left|V_{i 2}\right|^{2} \cos ^{2} \beta+\left|U_{i 2}\right|^{2} \sin ^{2} \beta .
\end{aligned}
$$

Following the notation of Ref. [12], we have introduced the neutralino mixing matrix $N$, the two chargino mixing matrices $U$ and $V$, the mixing angle $\alpha$ in the neutral $\mathrm{CP}-$ even Higgs sector, and the ratio of Higgs vacuum expectation values $\tan \beta$. The structure of eqs. (2) is easily understood, since gravitinos only couple to members of the same supermultiplet (in current basis). Each term is therefore the product of a higgsino component of a chargino or neutralino and the component of the corresponding Higgs current eigenstate in the relevant Higgs mass eigenstate.

It would appear from eq. (四) than the partial widths for Higgs to gravitino decays could be made arbitrarily large by making $m_{\widetilde{G}}$ very small, if $m_{\Phi}>m_{\tilde{\chi}_{i}}$. However, as stated earlier a very small $m_{\widetilde{G}}$ corresponds to a small SUSY breaking scale $\Lambda$. As argued in Ref. [2], present lower bounds on sparticle masses imply that $\Lambda$ should be several hundred $\mathrm{GeV}$ at least, which corresponds to $m_{\widetilde{G}}>$ (a few) $10^{-5} \mathrm{eV}$. We therefore chose $m_{\widetilde{G}}=10^{-4}$ $\mathrm{eV}$ in most of numerical examples. This corresponds to $\Lambda=650 \mathrm{GeV}$, which is probably quite close to its lower bound in realistic models. Note that $\Lambda^{2}$ is the vev of the largest $\mathrm{F}$ - or D-term in the model, which is usually (much) larger than the squared masses of sparticles in the visible sector.

Furthermore, if $m_{\widetilde{G}} \leq 1 \mathrm{eV}$, even the lightest sparticle in the visible sector will decay inside the detector [1, 2]. Here we make the usual assumption that the lightest neutralino $\tilde{\chi}_{1}^{0}$ is this next-to-lightest sparticle, the LSP being of course the gravitino. Associate $\tilde{\chi}_{1}^{0} \widetilde{G}$ production and $\tilde{\chi}_{1}^{0} \tilde{\chi}_{1}^{0}$ pair production at $e^{+} e^{-}$colliders then lead to final states with one or two isolated photons and missing energy-momentum. No significant excess over SM expectations has yet been observed in these channels. Following Refs. [3, 国 we interpret this as implying

$$
\begin{aligned}
\sigma\left(e^{+} e^{-} \rightarrow \widetilde{G} \tilde{\chi}_{1}^{0}\right)<0.1 \mathrm{pb} & \left(\sqrt{s}=91 \mathrm{GeV},\left|\cos \theta_{\gamma}\right| \leq 0.7\right) \\
\sigma\left(e^{+} e^{-} \rightarrow \tilde{\chi}_{1}^{0} \tilde{\chi}_{1}^{0}\right)<0.2 \mathrm{pb} & (\sqrt{s}=162 \mathrm{GeV}) .
\end{aligned}
$$

Expressions for these cross sections can be found in Refs. [3] and [13], respectively. In particular the LEP1 constraint (3a) is very restrictive [3], if $m_{\tilde{\chi}_{1}^{0}}<M_{Z}$.

Further constraints on the relevant parameter space can be derived from the negative outcome of chargino searches. Note that in our scenario chargino pair production always leads to large amounts of visible energy, since $\tilde{\chi}_{1}^{0}$ decays inside the detector. A small $\tilde{\chi}_{1}^{ \pm}-\tilde{\chi}_{1}^{0}$ mass difference is thus no longer problematic for these searches. We have therefore interpreted recent chargino searches at LEP as implying

$$
m_{\tilde{\chi}_{1}^{ \pm}}>80 \mathrm{GeV}
$$

In fact, in this scenario existing Tevatron data most likely imply a significantly stronger bound on the chargino mass. The authors of Ref. [2] estimate that the present data samples of the CDF and D0 collaborations should contain several additional events with two hard photons and substantial missing transverse momentum if $m_{\tilde{\chi}_{1}^{ \pm}} \leq 120$ to 130 
GeV. No such events have been observed [9]. Although no "official" experimental bound on $m_{\tilde{\chi}_{1}^{ \pm}}$has yet been published for the light gravitino scenario, it seems very likely that combinations of parameters giving $m_{\tilde{\chi}_{1}^{ \pm}}<120 \mathrm{GeV}$ are already excluded. We will therefore indicate the consequences of replacing the lower bound (田) by this more restrictive constraint.

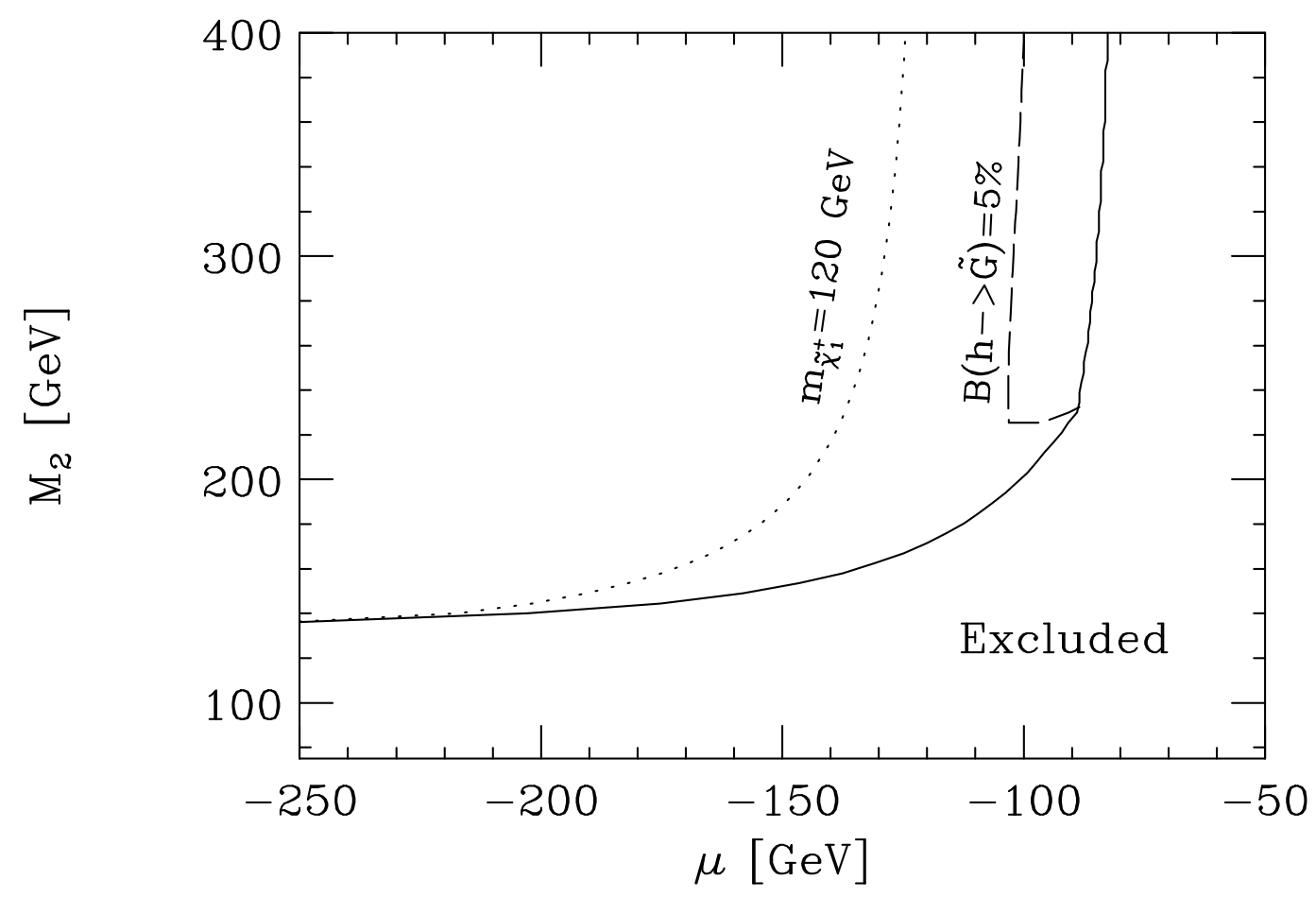

Figure 1: Contour of constant $B R\left(h \rightarrow \widetilde{G} \tilde{\chi}^{0}\right)=5 \%$ in the $\left(\mu, M_{2}\right)$ plane, for $m_{\tilde{t}_{L}}=$ $m_{\tilde{t}_{R}}=1 \mathrm{TeV}, A_{t}=\sqrt{6} \mathrm{TeV}, \tan \beta=50, m_{A}=500 \mathrm{GeV}$ and $m_{\widetilde{G}}=10^{-4} \mathrm{eV}$. The region to the right and below the solid line is excluded by the experimental bounds (3) and (4), where we have assumed $m_{\tilde{e}_{L}}=m_{\tilde{e}_{R}}=300 \mathrm{GeV}$. In the region to the right and below the dotted contour the mass of the lighter chargino is below $120 \mathrm{GeV}$; this region is most likely excluded by present Tevatron data, as discussed in the text.

We are now ready to present some numerical results. We begin with a discussion of the decays of the lighter neutral Higgs scalar $h$. Eq. (1) shows that $\Gamma\left(h \rightarrow \widetilde{G} \tilde{\chi}^{0}\right)$ will be maximal if $m_{h}$ is maximal. In Fig. 1 we have therefore chosen $\tan \beta=50$, a heavy pseudoscalar Higgs boson $m_{A}=500 \mathrm{GeV}$, large degenerate third generation squark masses $\left(m_{\tilde{t}_{L}}=m_{\tilde{t}_{R}}=1 \mathrm{TeV}\right)$, and sizable $\tilde{t}_{L}-\tilde{t}_{R}$ mixing $\left(A_{t}=\sqrt{6} \mathrm{TeV}\right)$. This maximizes [14] one-loop corrections to $m_{h}$, which have been computed using the formalism of Ref. [15]; we obtain $m_{h}=139 \mathrm{GeV}$ for our choice $m_{t}$ (pole) $=176 \mathrm{GeV}$.

*We include the leading 2-loop QCD corrections [16] by using the running top mass at scale $Q=\sqrt{m_{t} m_{\tilde{t}}}$ in the expressions for the corrected Higgs boson masses. However, we neglect electroweak corrections [17, which could reduce $m_{h}$ by several GeV. 
figures we assume that the $U(1)_{Y}$ and $S U(2)$ gaugino masses satisfy the "unification condition" $M_{1}=5 / 3 \tan ^{2} \theta_{W} M_{2} \simeq 0.5 M_{2}$. Relaxing this assumption will not change our results significantly, since we are interested in the higgsino-like neutralino and chargino states, see eqs. (21). Further, we assume $m_{\tilde{e}_{L}}=m_{\tilde{e}_{R}}=300 \mathrm{GeV}$. In this case the constraint (3b) is always weaker than the combination of the constraints (3a) and (4). Our calculation of the total decay widths of the MSSM Higgs bosons includes decays [18] into massive $f \bar{f}$ pairs $(f=c, \tau, b, t)$, loop decays into two gluons or two photons and, where appropriate, decays into $V V$ final states $\left(V=W^{ \pm}\right.$or $Z$, one of which may be off-shell) as well as decays of the heavy Higgs bosons into lighter ones $(H \rightarrow h h, A \rightarrow Z h)$.

The region to the right and below the solid line in Fig. 1 is excluded by present constraints; in the region of small $|\mu|$, the constraint (四) is the relevant one, while for larger $|\mu|$ (gaugino-like $\tilde{\chi}_{1}^{0}$ ) the condition (3a) is most restrictive. This still allows a narrow strip of parameter space where $B R\left(h \rightarrow \widetilde{G} \tilde{\chi}^{0}\right)$ exceeds $5 \%$, as indicated by the long-dashed contour. However, this region can be excluded in the likely case that existing Tevatron data already impose the limit $m_{\tilde{\chi}_{1}^{ \pm}} \geq 120 \mathrm{GeV}$; this bound is violated to the right and below the dotted contour. We therefore conclude that, even if a light gravitino exists, it cannot change the $h$ decay patterns significantly.

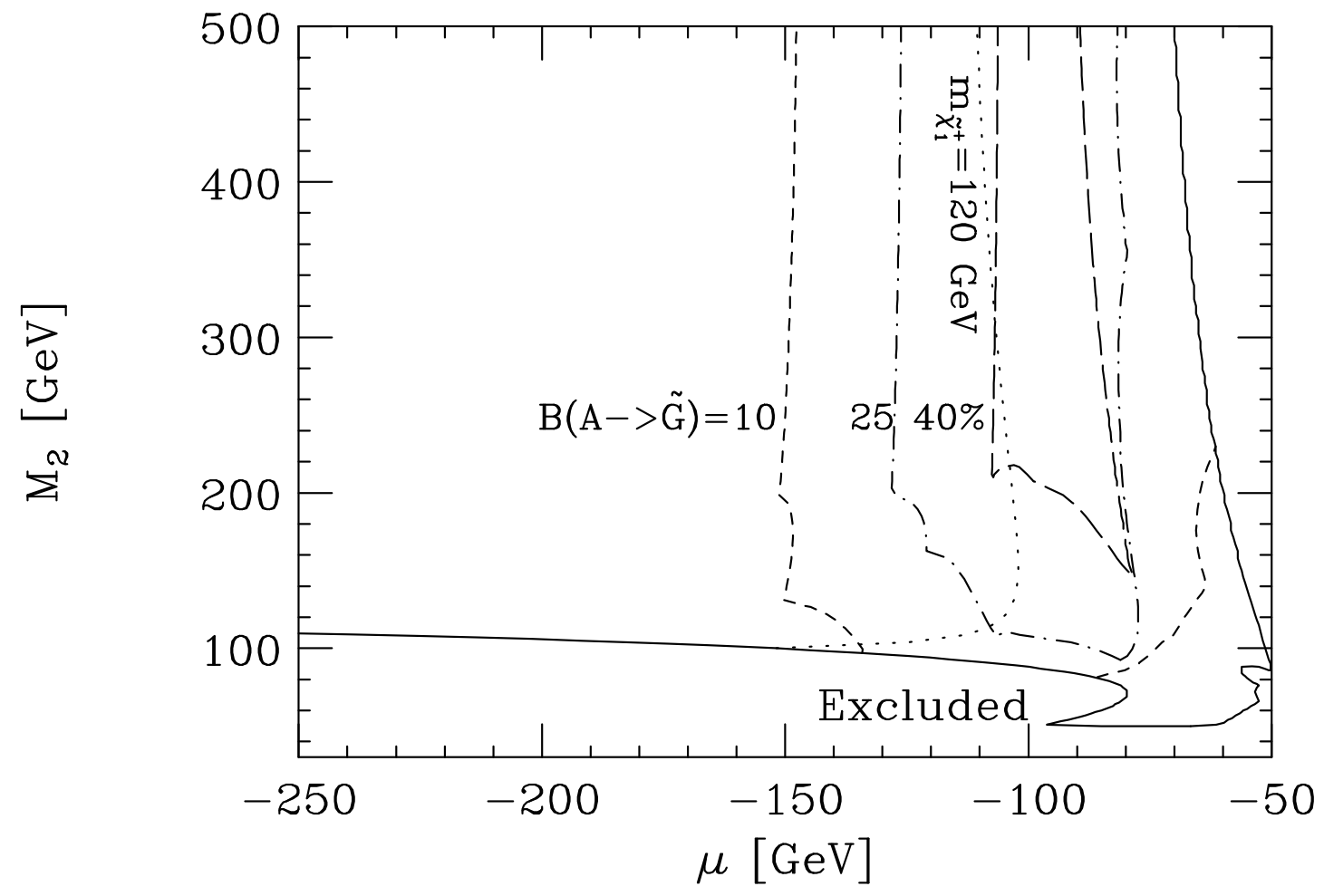

Figure 2: Contours of constant $B R\left(A \rightarrow \widetilde{G} \tilde{\chi}^{0}\right)$ in the $\left(\mu, M_{2}\right)$ plane, for $m_{A}=200 \mathrm{GeV}$ and $\tan \beta=1.5$. The sfermion and gravitino mass parameters are as in Fig. 1. The region to the right and below the solid line is excluded by the constraints (3) and (国), while to the right and below the dotted contour the lighter chargino mass is below $120 \mathrm{GeV}$. 
However, the situation could be quite different for the heavier Higgs bosons. As an example, in Fig. 2 we show contours of constant $B R\left(A \rightarrow \widetilde{G} \tilde{\chi}^{0}\right)$ for $\tan \beta=1.5$ and $m_{A}=200 \mathrm{GeV}$; the values of the other parameters are as in Fig. 1 . We have chosen a small value of $\tan \beta$ here in order to minimize the partial widths for $A \rightarrow b \bar{b}$ and $A \rightarrow \tau^{+} \tau^{-}$ decays. Since $m_{\tilde{t}}$ and $A_{t}$ are large, $m_{h} \simeq 100 \mathrm{GeV}$ so that $A \rightarrow Z h$ decays are phase space suppressed. We see that in this case the gravitino mode can account for $40 \%$ of all $A$ decays even if we require $m_{\tilde{\chi}_{1}^{ \pm}} \geq 120 \mathrm{GeV}$ (dotted contour). This is true even though we chose a rather moderate value of $m_{A}$ in this figure, so that $A H$ pairs can be produced at the proposed next linear $e^{+} e^{-}$collider operating at $\sqrt{s}=500 \mathrm{GeV}$.

Before we discuss $A H$ production in more detail, we explore how the results of Fig. 2 depend on the values of various parameters. Increasing $\tan \beta$ tends to reduce the importance of $A$ decays into sparticles, at least for $m_{A}<2 m_{t}$, since the partial widths into $b \bar{b}$ and $\tau^{+} \tau^{-}$pairs scale $\propto \tan ^{2} \beta$. Choosing the opposite sign of $\mu$ also reduces the maximal branching ratio for $A \rightarrow \widetilde{G} \tilde{\chi}^{0}$ decays. The partial widths for these decays do not change much when the sign of $\mu$ is flipped, but the constraint (đ) is more restrictive for $\mu>0$; in addition, the partial widths into pairs of neutralinos or charginos are enhanced. As a result, we find $B R\left(A \rightarrow \widetilde{G} \tilde{\chi}^{0}\right) \leq 25 \%$ for $\mu>0$, keeping all other parameters as in Fig. 2; this is reduced to $15 \%$ if we require $m_{\tilde{\chi}_{1}^{ \pm}} \geq 120 \mathrm{GeV}$.

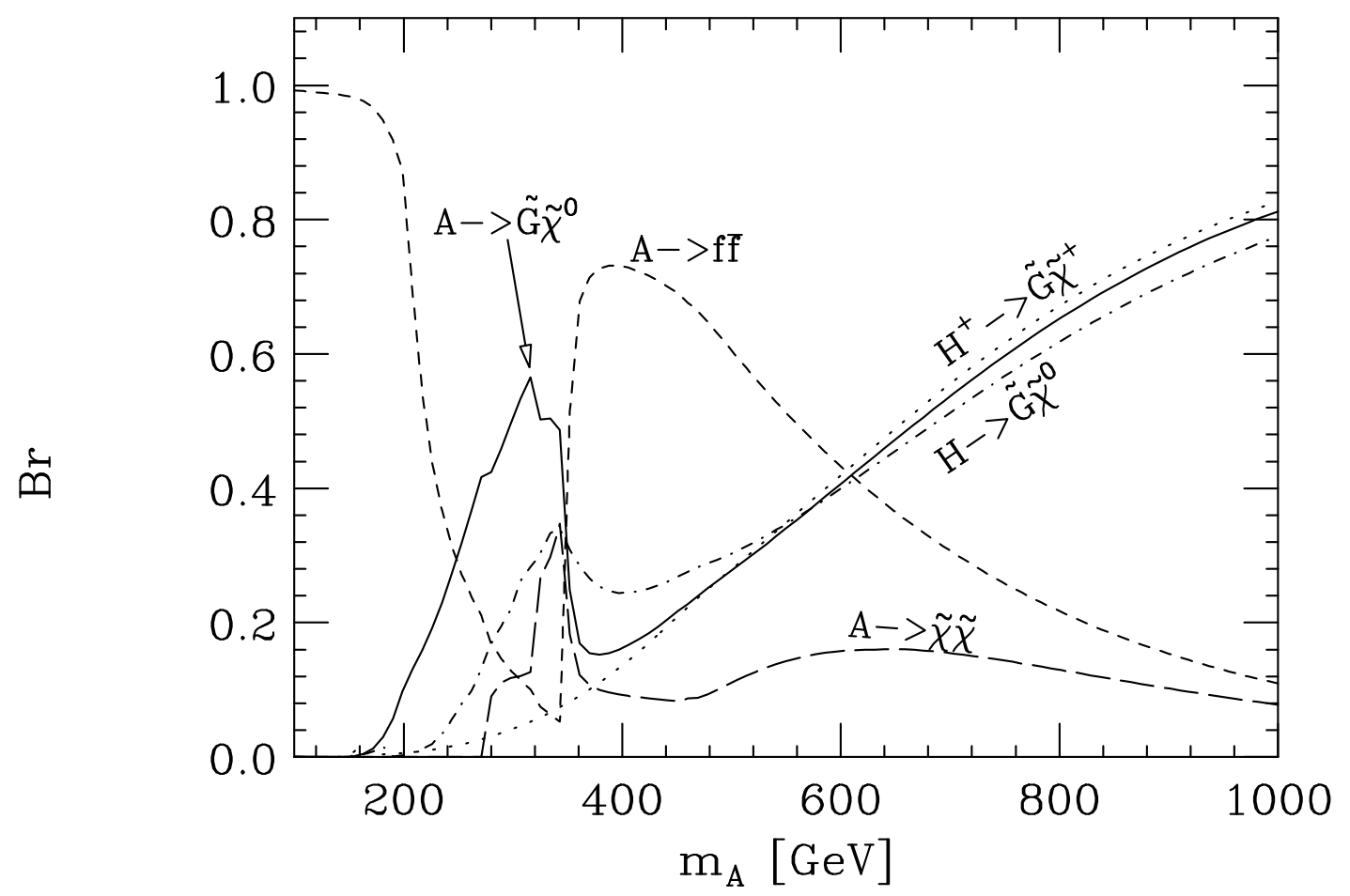

Figure 3: Branching ratios of the heavy Higgs bosons of the MSSM as a function of the mass of the pseudoscalar Higgs boson, for $M_{2}=300 \mathrm{GeV}, \mu=-150 \mathrm{GeV}, \tan \beta=$ $2, m_{\widetilde{G}}=10^{-4} \mathrm{eV}$ and the same sfermion mass parameters as in Fig. 1. Here $f$ stands for any SM quark or lepton, $\tilde{\chi}^{0}$ for any of the four neutralinos of the MSSM, and $\tilde{\chi}$ for any of the two charginos or four neutralinos. 
As discussed earlier, we probably cannot enhance the partial widths into gravitinos by reducing $m_{\widetilde{G}}$ even further, since this would give too small a SUSY breaking scale $\Lambda$. However, we saw in eq. (1) that these partial widths also rise very quickly with increasing Higgs boson mass. This is illustrated in Fig. 3, which shows some branching ratios of the heavy Higgs bosons of the MSSM as a function of $m_{A}$. Here we have chosen $M_{2}=300$ $\mathrm{GeV}, \mu=-150 \mathrm{GeV}$ and $\tan \beta=2$, corresponding to $m_{\tilde{\chi}_{1}^{0}}=136 \mathrm{GeV}$ and $m_{\tilde{\chi}_{1}^{ \pm}}=158$ $\mathrm{GeV}$. The parameters of the squark mass matrices are as in Figs. 1 and 2. We see that, in spite of the $m_{A}^{5}$ factor, the branching ratio for $A \rightarrow \widetilde{G} \tilde{\chi}^{0}$ decays at first grows rather slowly from the threshold at $m_{A}=m_{\tilde{\chi}_{1}^{0}}$, due to the kinematical factor $\left(1-m_{\tilde{\chi}}^{2} / m_{A}^{2}\right)^{4}$ in eq. (11). This is to be contrasted with the rapid increase of the branching ratio into heavy SM fermions just beyond the $t \bar{t}$ threshold (short dashed curve); here the threshold factor is $\left(1-4 m_{t}^{2} / m_{A}^{2}\right)^{1 / 2}$. Finally, the long dashed curve shows the branching ratio for $A$ decays into pairs of neutralinos or charginos, which exhibits several thresholds. Together with the onset of $A \rightarrow t \bar{t}$ decays, these supersymmetric decay modes at first reduce $B R\left(A \rightarrow \widetilde{G} \tilde{\chi}^{0}\right)$ as $m_{A}$ is increased beyond $320 \mathrm{GeV}$. These $\tilde{\chi} \tilde{\chi}$ modes are also responsible for the reduced importance of the gravitino modes at small $|\mu|$ shown in Fig. 2. However, eventually the $m_{A}^{5}$ factor wins out, causing the solid curve to rise again as $m_{A}$ is increased beyond 400 $\mathrm{GeV}$.

So far we have only discussed decays of $h$ and $A$. For large $m_{A}, m_{A}^{2} \gg \mu^{2}$, the partial width for $H \rightarrow \widetilde{G} \tilde{\chi}^{0}$ decays is very similar to that for $A \rightarrow \widetilde{G} \tilde{\chi}^{0}$ decays. However, if $\tan \beta$ is not large, $H$ can also have a sizable branching ratio into $h h$ pairs 18. As shown by the dot-dashed curve in Fig. 3, this reduces the branching ratio for decays of the heavy neutral scalar into gravitinos. In fact, for the parameters of Fig. 2 this mode completely dominates $H$ decays, leading to $B R\left(H \rightarrow \widetilde{G} \tilde{\chi}^{0}\right)<2 \%$ in this case. On the other hand, for large $m_{A}$ the charged Higgs boson has an even slightly larger branching ratio into the gravitino mode than the pseudoscalar (dotted curve). However, at small $m_{A}$, the branching ratio for $H^{+} \rightarrow \widetilde{G} \tilde{\chi}^{+}$decays is small, partly because the lightest chargino is heavier than the lightest neutralino, and partly because the threshold for $H^{+} \rightarrow t \bar{b}$ decays is at much lower values of $m_{A}$ than that for $A \rightarrow t \bar{t}$ decays. It should be emphasized that decays into light gravitinos could dominate the decays of all three heavy Higgs bosons of the MSSM, if $m_{A} \geq 700 \mathrm{GeV}$. Note also that for our choice $m_{\widetilde{G}}=10^{-4} \mathrm{eV}$, the total decay width of these Higgs bosons is around $100 \mathrm{GeV}$ for $m_{A}=1 \mathrm{TeV}$.

Finally, while it is unlikely that $m_{\widetilde{G}}$ is significantly smaller than $10^{-4} \mathrm{eV}$, it could certainly be larger. We find that for $m_{\widetilde{G}}=5 \cdot 10^{-4} \mathrm{eV}, B R\left(A \rightarrow \widetilde{G} \tilde{\chi}^{0}\right)<15 \%$ for $m_{A} \leq 1$ $\mathrm{TeV}$. Clearly this is about the largest value of $m_{\widetilde{G}}$ where this branching ratio can be sizable, if $m_{A}$ lies in the range indicated by naturalness arguments.

\section{Collider Signals}

The signature for $A \rightarrow \widetilde{G} \tilde{\chi}^{0}$ decays depends on which neutralino states are produced in association with the light gravitino. Recall that these decays proceed through the higgsino components of the neutralinos. In most cases two of the four neutralinos of the MSSM are higgsino-like and the other two are gaugino-like, although occasionally two states are strongly mixed. If the higgsino-dominated states are the heavier neutralinos 
$\tilde{\chi}_{3,4}^{0}, A \rightarrow \widetilde{G} \tilde{\chi}^{0}$ decays will lead to the subsequent cascade decays $\tilde{\chi}_{3,4}^{0} \rightarrow \tilde{\chi}_{1,2}^{0} f \bar{f}$, where $f$ stands for any kinematically accessible quark or lepton; these decays proceed through (virtual or real) $h, Z$ and $\tilde{f}$ exchange. $\tilde{\chi}_{3,4}^{0} \rightarrow \tilde{\chi}_{1}^{ \pm} f \bar{f}^{\prime}$ decays may also occur. This can lead to quite complicated final states. However, in this scenario some decays of the type $A \rightarrow \tilde{\chi}_{1,2} \tilde{\chi}_{3,4}$ are probably also allowed. Since they combine a higgsino-like and a gaugino-like state, the relevant couplings are large [12]. In this case $A \rightarrow \widetilde{G} \tilde{\chi}^{0}$ decays are therefore only important if $m_{A}$ is quite large, probably beyond the reach of next generation colliders.

We therefore focus on the scenario where the higgsino-like states are lighter than the gaugino-like states. We saw in Fig. 2 that in such a scenario the branching ratio for $A \rightarrow \widetilde{G} \tilde{\chi}^{0}$ decays can be substantial already for $m_{A}=200 \mathrm{GeV}$. Note that the mass splitting between the higgsino-like states is usually quite small. $A \rightarrow \widetilde{G} \tilde{\chi}_{2}^{0}$ decays therefore differ from $A \rightarrow \widetilde{G} \tilde{\chi}_{1}^{0}$ decays only by a rather soft $f \bar{f}$ pair. With the possible exception $f=\mu$, such soft pairs are probably not detectable at all at hadron colliders like the planned LHC. At these colliders the most important Higgs production mechanism is gluon fusion, $g g \rightarrow h, H, A$. The signature for $A \rightarrow \widetilde{G} \tilde{\chi}^{0}$ decays would then be a single hard photon from $\tilde{\chi}_{1}^{0} \rightarrow \gamma \widetilde{G}$ and missing transverse momentum. Note that there is no Jacobian peak in the $p_{T}(\gamma)$ distribution, since the photon is only produced in a secondary decay. Unfortunately, this final state will probably suffer from considerable backgroundsin. Recall also that in this scenario all SUSY processes give rise to two hard photons and missing $p_{T}$ [2, 10]. They can therefore also contribute to the background if one of the photons is lost.f A detailed study will be necessary to decide whether vetoing the presence of additional jets and/or leptons will be sufficient to overcome this potentially very large background.

As usual, things should be much "cleaner" at $e^{+} e^{-}$colliders, especially if $A$ is light enough to be produced at a $500 \mathrm{GeV}$ collider. The main $A$ production mechanism at $e^{+} e^{-}$colliders is associate $A H$ production. We saw in Sec. 2 that if $m_{A} \leq 220 \mathrm{GeV}$, so that the cross section for $A H$ production is sizable at a $500 \mathrm{GeV}$ collider, and if $\tan \beta$ is relatively small, so that $B R\left(A \rightarrow \widetilde{G} \tilde{\chi}^{0}\right)$ can be substantial, then $B R\left(H \rightarrow \widetilde{G} \tilde{\chi}^{0}\right)$ is very small, largely due to $H \rightarrow h h$ decays. $A H$ production and subsequent $A \rightarrow \widetilde{G} \tilde{\chi}^{0}$ decay would then most often lead to a final state with two $b \bar{b}$ pairs from the decays of the two light Higgs scalars, a hard isolated photon, and perhaps a soft $f \bar{f}$ pair from $\tilde{\chi}_{2}^{0} \rightarrow \tilde{\chi}_{1}^{0} f \bar{f}$ decays. There should be little background in this channel, especially if one requires several $b$-tags. Furthermore, events of this type should allow to determine the masses of all involved (s)particles by studying kinematic distributions. Clearly $m_{h}$ can be obtained from $b \bar{b}$ invariant mass distributions. Combinatorial backgrounds can be reduced by choosing the pairing that gives the most similar values for the two $b \bar{b}$ invariant masses. Of course, $m_{h}$ can also be obtained completely independently from $e^{+} e^{-} \rightarrow Z h$ events in

\footnotetext{
${ }^{\dagger}$ For instance as irreducible backgrounds, one has $\gamma Z$ production and subsequent $Z \rightarrow \nu \bar{\nu}$ decay; $W \gamma$ final states followed by $W \rightarrow l \nu_{l}(l=e, \mu, \tau)$ decays can also contribute to the background if the lepton is not detected.

${ }^{\ddagger}$ For $m_{\widetilde{G}}=10^{-4} \mathrm{eV}, \tilde{q} \rightarrow \widetilde{G} q$ decays will dominate even over $\tilde{q} \rightarrow \tilde{g} q$ decays if $m_{\tilde{q}}>800 \mathrm{GeV}$ [2]; similarly, gluinos will dominantly decay into a gluon and a gravitino if $m_{\widetilde{G}} \leq 10^{-3} \mathrm{eV} \cdot\left(m_{\tilde{q}} / 1 \mathrm{TeV}\right)^{2}$. These decays lead to final states with rather low jet multiplicity, increasing the probability that the jets escape detection.
} 
this scenario; the value of $m_{h}$ derived from the kind of $A H$ events we are studying here would therefore be a cross-check on the interpretation of the observed events.

In principle one can get $m_{H}$ from the invariant mass of the $4 b$ system, and $m_{A}$ as the mass recoiling against this system. However, the $b \rightarrow c \rightarrow s$ cascade decays of the four $b$ (anti)quarks are likely to produce some neutrinos, which will smear out these invariant mass distributions. Once $m_{h}$ is known, one might be able to at least partly correct for this by using constraint fits. Alternatively, one can obtain $m_{A}$ and $m_{H}$, as well as $m_{\tilde{\chi}_{1}^{0}}$, from the photon energy spectrum. Two examples of this spectrum are shown in Fig. 4, for $m_{A}=200 \mathrm{GeV}, m_{H}=220 \mathrm{GeV}, \sqrt{s}=500 \mathrm{GeV}$ and $m_{\tilde{\chi}_{1}^{0}}=100 \mathrm{GeV}$ (solid) and 150 $\mathrm{GeV}$ (dashed). Here we have treated $\widetilde{G} \tilde{\chi}_{2}^{0}$ final states the same as $\widetilde{G} \tilde{\chi}_{1}^{0}$ final states; the additional soft $f \bar{f}$ pair does not distort the $E_{\gamma}$ spectrum significantly.

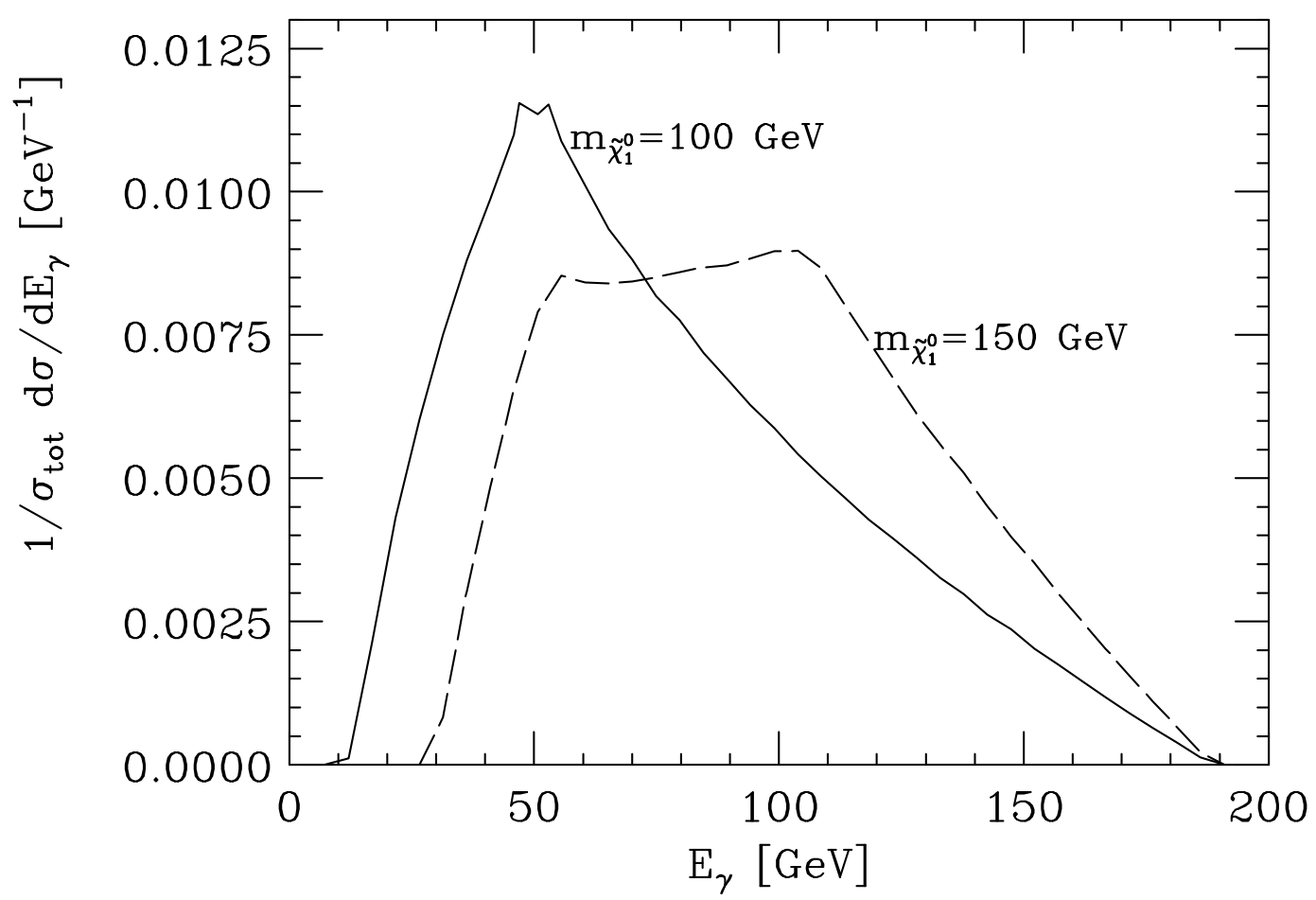

Figure 4: The photon energy spectrum in the lab frame for events of the type $e^{+} e^{-} \rightarrow H A$ with subsequent $A \rightarrow \widetilde{G} \tilde{\chi}_{1}^{0}$ decay and $\tilde{\chi}_{1}^{0} \rightarrow \widetilde{G} \gamma$. We have taken $m_{H}=220 \mathrm{GeV}, m_{A}=200$ $\mathrm{GeV}, \sqrt{s}=500 \mathrm{GeV}$, and two different values of the $\tilde{\chi}_{1}^{0}$ mass, as indicated. The acceptance cut $\left|\cos \theta_{\gamma}\right| \leq 0.9$ has been applied.

We see that the photons are always quite hard; an acceptance cut like $E_{\gamma}>10 \mathrm{GeV}$ would therefore not reduce the signal at all. In Fig. 4 we have required $\left|\cos \theta_{\gamma}\right| \leq 0.9$, where $\theta_{\gamma}$ is the angle between the outgoing photon and the $e^{-}$beam direction in the lab frame. This reduces the accepted cross section by some $8 \%$. Recall that $d \sigma\left(e^{+} e^{-} \rightarrow\right.$ $A H) / d \cos \theta_{A} \propto \sin ^{2} \theta_{A}$, where $\theta_{A}$ is the angle between the pseudoscalar Higgs boson and 
the $e^{-}$beam; the distribution in $\cos \theta_{\gamma}$ is therefore mildly peaked at $\cos \theta_{\gamma}=0$.

Of course, this angular acceptance cut has no bearing on the endpoints of the photon energy spectrum, which are at

$$
E_{\gamma}^{\min , \max }=\frac{1}{2}\left[E_{\max }\left(\tilde{\chi}_{1}^{0}\right) \pm p_{\max }\left(\tilde{\chi}_{1}^{0}\right)\right]
$$

where $E_{\max }\left(\tilde{\chi}_{1}^{0}\right)$ is the maximal energy of the neutralino in the lab frame and $p_{\max }\left(\tilde{\chi}_{1}^{0}\right)=$ $\sqrt{E_{\max }^{2}\left(\tilde{\chi}_{1}^{0}\right)-m_{\tilde{\chi}}^{2}}$ is the absolute value of the corresponding $3-$ momentum. Since $A \rightarrow \widetilde{G} \tilde{\chi}^{0}$ decays are isotropic, the distribution in $E\left(\tilde{\chi}_{1}^{0}\right)$ is flat, with endpoints

$$
E_{\max , \min }\left(\tilde{\chi}_{1}^{0}\right)=\frac{1}{m_{A}}\left[E_{(A)}\left(\tilde{\chi}_{1}^{0}\right) \pm k E_{(A)}(\widetilde{G})\right]
$$

Here,

$$
E_{(A)}\left(\tilde{\chi}_{1}^{0}\right)=\frac{m_{A}^{2}+m_{\tilde{\chi}}^{2}}{2 m_{A}}, \quad E_{(A)}(\widetilde{G})=\frac{m_{A}^{2}-m_{\tilde{\chi}}^{2}}{2 m_{A}}
$$

are the neutralino and primary gravitino energy in the $A$ rest frame; $E_{A}=\left(s+m_{A}^{2}-\right.$ $\left.m_{H}^{2}\right) /(2 \sqrt{s})$ is the energy of $A$ in the lab frame and $k=\sqrt{E_{A}^{2}-m_{A}^{2}}$ is the absolute value of the 3-momentum of $A$ (or $H$ ) in the lab frame.

The determination of the endpoints of the photon energy spectrum already gives two constraints on the three unknowns $m_{A}, m_{H}$ and $m_{\tilde{\chi}_{1}^{0}}$. In addition, Fig. 4 shows that the photon energy spectrum is almost flat over some range of energies; indeed, it would be completely flat had we not imposed the acceptance cut $\left|\cos \theta_{\gamma}\right| \leq 0.9$. This is due to the fact that both the $A \rightarrow \widetilde{G} \tilde{\chi}^{0}$ decay and the $\tilde{\chi}_{1}^{0} \rightarrow \widetilde{G} \gamma$ decay are isotropic in their respective rest frames. In the absence of acceptance cuts this leads to a flat spectrum between the points

$$
E_{\gamma}^{ \pm}=\frac{1}{2}\left[E_{\min }\left(\tilde{\chi}_{1}^{0}\right) \pm p_{\min }\left(\tilde{\chi}_{1}^{0}\right)\right]
$$

where $E_{\min }\left(\tilde{\chi}_{1}^{0}\right)$ has been given in eq. (6). The solid curve in Fig. 4 shows that this plateau can be very narrow. This happens for combinations of parameters leading to a small value of $p_{\min }\left(\tilde{\chi}_{1}^{0}\right)$; in the given case this quantity amounts to just $6 \mathrm{GeV}$. In more generic cases, one should be able to determine both $E_{\gamma}^{+}$and $E_{\gamma}^{-}$with good precision, given sufficient statistics. This would give two additional, independent constraints on three unknown masses.

Another possibly useful kinematic distribution is that in the invariant mass of the two gravitinos produced in the $A \rightarrow \widetilde{G} \tilde{\chi}^{0} \rightarrow \widetilde{G} \widetilde{G} \gamma$ decay chain. Since the decays are isotropic, one simply finds $d \sigma / d M_{\widetilde{G} \widetilde{G}} \propto M_{\widetilde{G} \widetilde{G}}$ with a sharp cut-off at $M_{\widetilde{G} \widetilde{G}}^{\max }=\sqrt{m_{A}^{2}-m_{\tilde{\chi}}^{2}}$. However, the $\widetilde{G} \widetilde{G}$ invariant mass will be equal to the missing mass only if there are no additional sources of missing energy in the event (neutrinos from the decay of $b$ quarks, or initial state radiation that goes down one of the beam pipes). 


\section{Summary and Conclusions}

In this note we have studied the decays of MSSM Higgs bosons into light gravitinos. We found that present experimental bounds already exclude the possibility that such decays are relevant for the light neutral Higgs scalar, but they could be quite important, or even dominant, for the heavier Higgs bosons if $m_{\widetilde{G}} \leq 0.5 \mathrm{meV}$. In the simplest case this gives rise to final states with a hard isolated photon and missing (transverse) energy. It is doubtful whether this signal is detectable at the LHC, but associate $A H$ production at $e^{+} e^{-}$colliders will have very little background if only $A$ decays into a gravitino and a neutralino while $H$ decays into a fully reconstructable final state. We argued that such a scenario is in fact likely if $A$ is sufficiently light to be produced at a $500 \mathrm{GeV}$ collider. We also showed that in this favorable situation one can in principle reconstruct $m_{H}, m_{A}$ and $m_{\tilde{\chi}_{1}^{0}}$ from the energy spectrum of the photon.

In practice it will probably be better to reconstruct the neutralino mass directly from neutralino pair events. Since the neutralino must have a substantial higgsino component if the branching ratio for $A \rightarrow \widetilde{G} \tilde{\chi}^{0}$ decays is sizable, the cross section for neutralino pair production at the same $e^{+} e^{-}$collider will be quite substantial - in fact, significantly larger than the $A H$ production cross section. This also implies that one need not worry about Higgs decays into gravitinos if one does not find sizable signals for neutralino and chargino

pair production at the same energy. In this respect Higgs decays into light gravitinos are quite similar to Higgs decays into pairs of neutralinos or charginos. At present, however, all these possibilities must be kept in mind when deriving search strategies for Higgs bosons at future colliders.

Acknowledgements: M.D. thanks the members of the LPM at the Université de Montpellier for their hospitality during a visit where this project was initiated.

\section{References}

[1] P. Fayet, Phys. Lett. 70B, 461 (1977); 86B, 272 (1979); and B175, 471 (1986).

[2] S. Ambrosiano, G.L. Kane, G.D. Kribs, S.P. Martin and S. Mrenna, Phys. Rev. D54, 5395 (1996).

[3] J.L. Lopez, D.V. Nanopoulos and A. Zichichi, hep-ph/9611437.

[4] J. Ellis, J.L. Lopez and D.V. Nanopoulos, hep-ph/9610470.

[5] M. Dine, A. Nelson and Y. Shirman, Phys. Rev. D51, 1362 (1995).

[6] J. Ellis, K. Enqvist and D.V. Nanopoulos, Phys. Lett. B147, 99 (1984), and B151, 357 (1985); J.L. Lopez and D.V. Nanopoulos, hep-ph/9607220, to appear in Mod. Phys. Lett.; J.L. Lopez, D.V. Nanopoulos and A. Zichichi, hep-ph/9609524.

[7] S. Park for the CDF collab., Search for new phenomena in CDF, talk presented at the 10th Topical Workshop on Proton Antiproton Collider Physics, R. Raha and G. Yodh, eds. 
[8] S. Dimopoulos, M. Dine, R. Raby and S. Thomas, Phys. Rev. Lett. 76, 3494 (1996); S. Ambrosiano, G.L. Kane, G.D. Kribs, S.P. Martin and S. Mrenna, Phys. Rev. Lett. 76, 3498 (1996).

[9] D0 collab., S. Adachi et al., Phys. Rev. Lett. 78, 2070 (1997); D. Toback for the CDF collab., talk presented at the conference of the Division of Particles and Fields of the American Physical Society, Minneapolis, MN, August 1996.

[10] H.A. Baer, M. Brhlik, C.-H. Chen and X.R. Tata, Phys. Rev. D55, 4463 (1997).

[11] R. Casalbuoni, S. DeCurtis, D. Dominici, F. Feruglio and R. Gatto, Phys. Lett. B215, 313 (1988), and Phys. Rev. D39, 2281 (1989).

[12] J.F. Gunion and H.E. Haber, Nucl. Phys. B272, 1 (1986).

[13] A. Bartl, H. Fraas and W. Majerotto, Nucl. Phys. B278, 1 (1986).

[14] Y. Okada, M. Yamaguchi and T. Yanagida, Phys. Lett. B262, 54 (1991).

[15] J. Ellis, G. Ridolfi and F. Zwirner, Phys. Lett. B257, 83 (1991), and B262, 477 (1991).

[16] R. Hempfling and A.H. Hoang, Phys. Lett. B331, 99 (1994).

[17] D.M. Pierce, A. Papadopoulos and S. Johnson, Phys. Rev. Lett. 68, 3678 (1992).

[18] For a review, see A. Djouadi, Int. J. Mod. Phys. A10, 1 (1995). 